\title{
Top pair production measurements, inclusive and differential
}

\author{
Javier Fernández Menéndez ${ }^{1}$ \\ Universidad de Oviedo \\ C/ Federico García Lorca s/n, Oviedo, Spain \\ E-mail: Javier.Fernández@cern.ch
}

Recent results and state-of-the-art on top-quark pair production cross sections in both inclusive and differential measurements are presented, obtained using data collected with the ATLAS, CMS and LHCb experiments during the LHC Run1 and Run2 periods up to the year 2016 at centre-ofmass energies ranging from 5.02 to $13 \mathrm{TeV}$. Results are confronted against SM NLO and NNLO order predictions, several MC generators and tunes, and parton density function variations, with a special focus on the latest Run2 results.

Sixth Annual Conference on Large Hadron Collider Physics (LHCP2018)

4-9 June 2018

Bologna, Italy

\footnotetext{
${ }^{1}$ Speaker, on behalf of the ATLAS, CMS and LHCb collaborations 


\section{Introduction}

The enormous datasets provided and the high production rate of top quark pairs (t $\mathrm{t} \overline{\mathrm{t}})$ at the LHC allow not only accurate inclusive cross section determinations but also precise measurements of differential and double-differential cross sections as a function of kinematic variables of the top quark and the $t \bar{t}$ system. As the higher order corrections to $t \bar{t}$ production are large, these measurements test the predictive power of perturbative QCD calculations and electroweak corrections. Similarly, $t \bar{t}$ observables at particle level can constrain the parton shower and hadronisation modelling. Due to the large mass of the top quark, $t \bar{t}$ cross sections are sensitive to the parton distribution function (PDF) of the gluon. Hence, these measurements have the potential to reduce the PDF uncertainties. Moreover, the generation of $t \bar{t}$ events requires a realistic modeling of parton showers. Measurements of kinematic properties and multiplicities of jets associated with $\mathrm{t} \overline{\mathrm{t}}$ production allow a detailed comparison of different parton shower models with the data and provide insight into their tuning.

Extensive measurements were performed by the ATLAS [1] and CMS [2] experiments at different proton-proton center-of-mass energies ranging from 5.02 to $13 \mathrm{TeV}$ and even in protonnucleus $(\mathrm{Pb})$ collisions for various $t \bar{t}$ decay channels. Recently, the LHCb [3] experiment has also

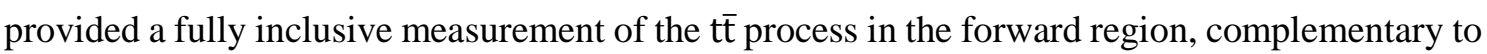
those by ATLAS and CMS. Results shown in this document correspond to data taken during the Run1 (at $\sqrt{s}_{\mathbf{s}}=7$ and $8 \mathrm{TeV}$ ) and Run2 (at $\sqrt{s}_{\mathbf{s}}=13 \mathrm{TeV}$ ) periods up to the year 2016.

\section{Inclusive cross section measurements}

With full next-to-next-to-leading order plus next-to-next-leading logarithmic order

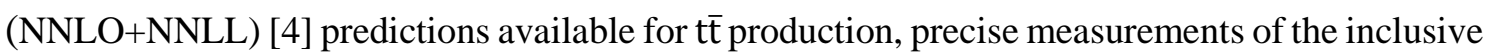
cross section are important tests for the underlying theory. The $t \bar{t}$ pair decays allow an event selection based on the topology of the particular decay of the W bosons as shown in Figure 1. There has been an excellent progress both on the theory and experiment side as shown in Figure 2 , with an impressive agreement on the inclusive cross-section up to now where the experimental uncertainty is comparable to the theoretical one. A special remark must be made to the latest inclusive additions: the ATLAS inclusive and fiducial lepton+jets measurements at $8 \mathrm{TeV}$ [5] with precisions of $\approx 6 \%$ and $4.5 \%$ respectively; and the CMS inclusive measurement at $5.02 \mathrm{TeV}$ using the dilepton and lepton+jets channels [6], with a precision of $12 \%$ given the low luminosity (27.4 $\mathrm{pb}^{-1}$ ) cumulated in the year 2015 at that centre-of-mass energy. This latter study at $5.02 \mathrm{TeV}$ represents complementary data which serve as reference for future $\mathrm{Pb}-\mathrm{Pb}$ and $\mathrm{p}-\mathrm{Pb}$ measurements, like the CMS observation of $t \bar{t}$ production in proton-nucleus (Pb) collisions [7], which constitutes a novel and theoretically precise probe of the nuclear gluon density at high virtualities.

Moreover, the LHCb experiment extended its fiducial cross section measurement at $8 \mathrm{TeV}$ performed in the lepton+b-jets channel in the forward $2.2<|\eta|<4.2$ region [8] to the dilepton $\mu \mathrm{eb}$ channel at $13 \mathrm{TeV}$ to the $2.0<|\eta|<4.5$ region using $1.93 \mathrm{fb}^{-1}$ of data collected in 2015 and 2016 [9]. The overall precision is $20 \%$ and it is statistically limited, but data from the years 2017 and 2018 are expected to improve the sensitivity in further studies. These LHCb measurements represent a unique and complementary study to the forward regions not accessible by ATLAS and CMS. 

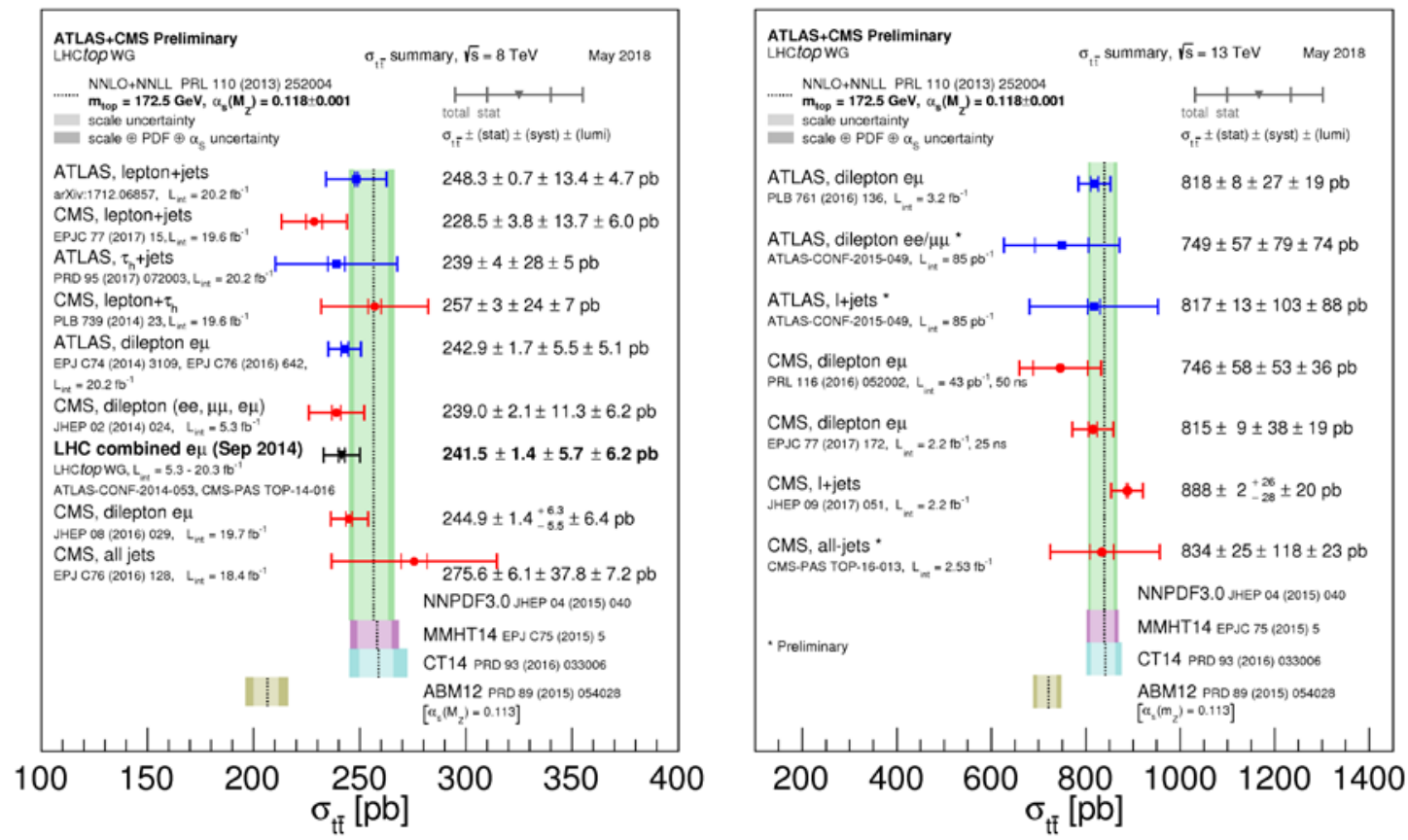

Figure 1. Summary of the ATLAS+CMS tt̄ inclusive cross section $8 \mathrm{TeV}$ (left) and $13 \mathrm{TeV}$ (right) measurements.

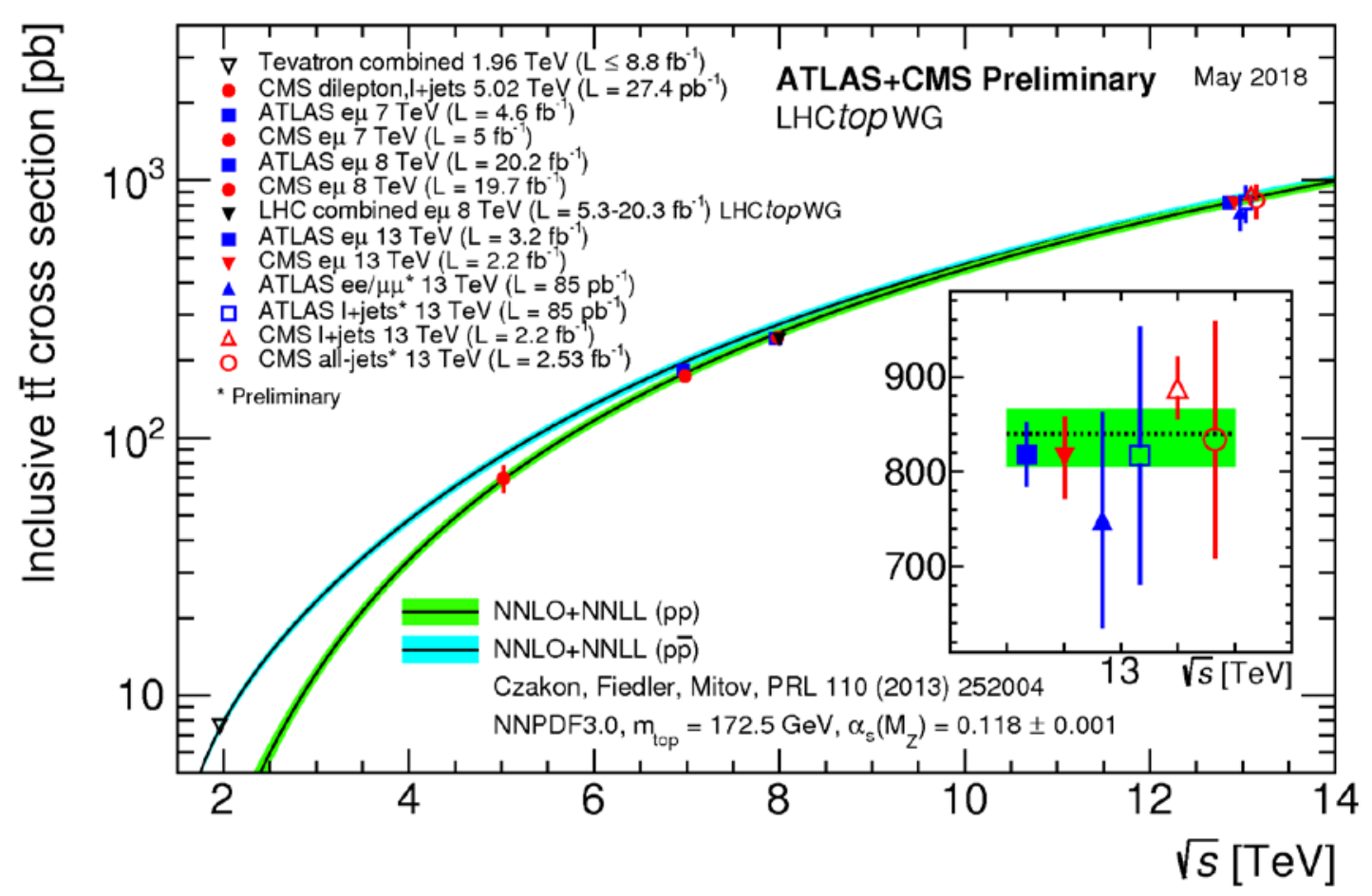

Figure 2. Evolution of the LHC t⿱一𫝀 inclusive cross section measurements with $\sqrt{s}$. Theory band represents uncertainties due to renormalization and factorization scale, parton density functions and the strong coupling. 


\section{Differential cross section measurements.}

Since Run1 there has been an ongoing discrepancy in full next-to-leading order (NLO) modelling of the top and $t \bar{t}$ system, most famously in the top quark transverse momentum $\mathrm{p}_{\mathrm{T}}(\mathrm{t})$. It has been observed in many channels, analyses, fiducial and full phase-space data, where only NNLO predictions are able to reproduce data, but those predictions are available at parton level only (see Figure 3). New results confirm this discrepancy and provide more precision including even electroweak corrections. The bottom line is that, so far, different full NLO Monte Carlo (MC) setups can describe some, but not all of the "standard" top and tt kinematics simultaneously.

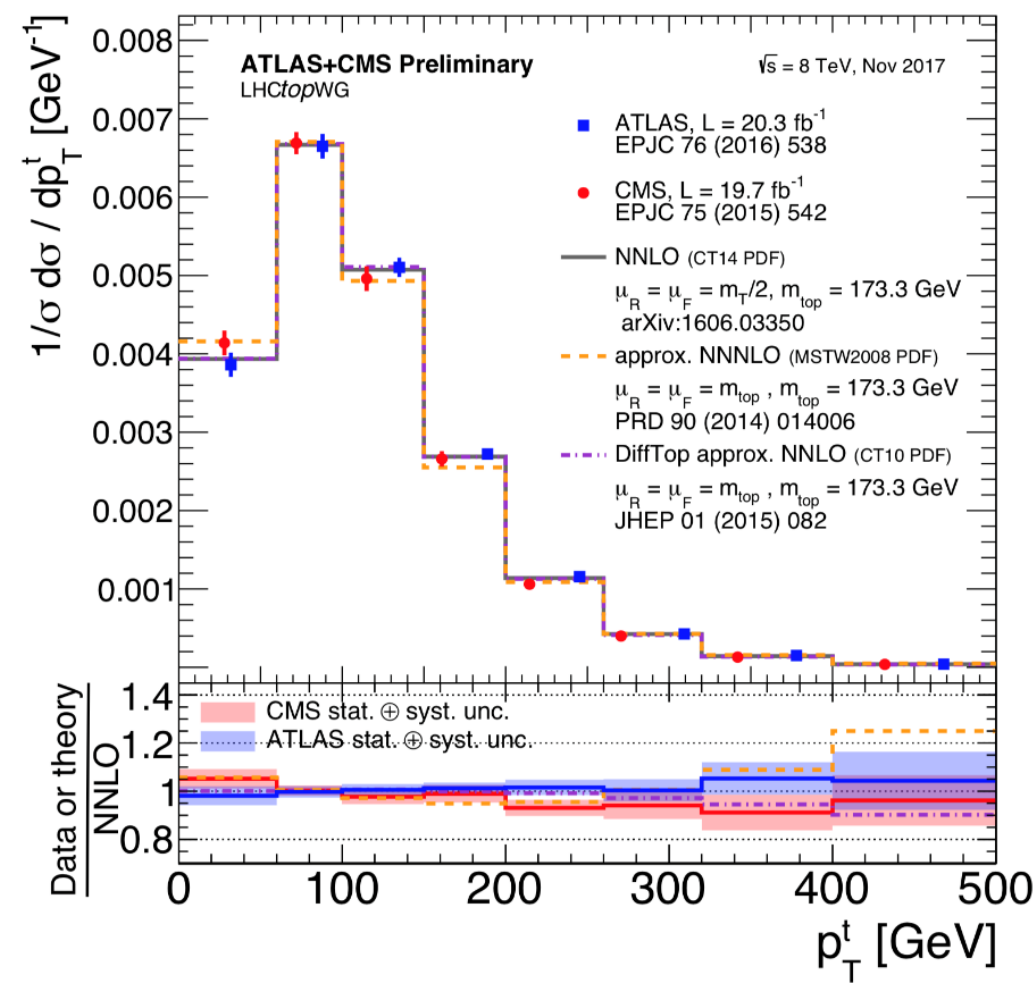

Figure 3. Full phase-space normalised differential $t \bar{t}$ cross section as a function of the transverse momentum of the top quark. The CMS and ATLAS results are compared to NNLO and approximate NNLO calculations. Both the CMS and ATLAS measurements are performed assuming a top quark mass value of $172.5 \mathrm{GeV}$. The shaded bands show the total uncertainty on the data measurements in each bin. The lower panel shows the ratio of the data measurements and the approximate NNLO calculations to the full NNLO calculation.

At present the latest differential result from Run2 comes from CMS [8] using year 2016 data and the clean $\mathrm{e} \mu$ channel, providing differential measurements both at parton level in the full phase space and at particle level within a phase space close to the experimental acceptance, the so called fiducial phase-space. Both ATLAS and CMS have chosen as default MC generator Powheg V2 [9] interfaced with Pythia8.2 [10] for the parton shower part for the Run2. This MC provides a reasonable agreement except in the top quark direct observables like $\mathrm{p}_{\mathrm{T}}(\mathrm{t})$ (see Figure 4), and the transverse momentum $\mathrm{p}_{\mathrm{T}}(\mathrm{t} \overline{\mathrm{t}})$ and invariant mass $\mathrm{m}(\mathrm{t} \overline{\mathrm{t}})$ of the $\mathrm{t} \overline{\mathrm{t}}$ system. Herwig++ [11] seems to improve the agreement but only at particle level. Moreover, NLO predictions at parton level show a slightly better agreement but not within uncertainties in the normalized distributions (see Figure 5). A previous equivalent e $\mu$ Run2 result from ATLAS [12] shows the same trend. 


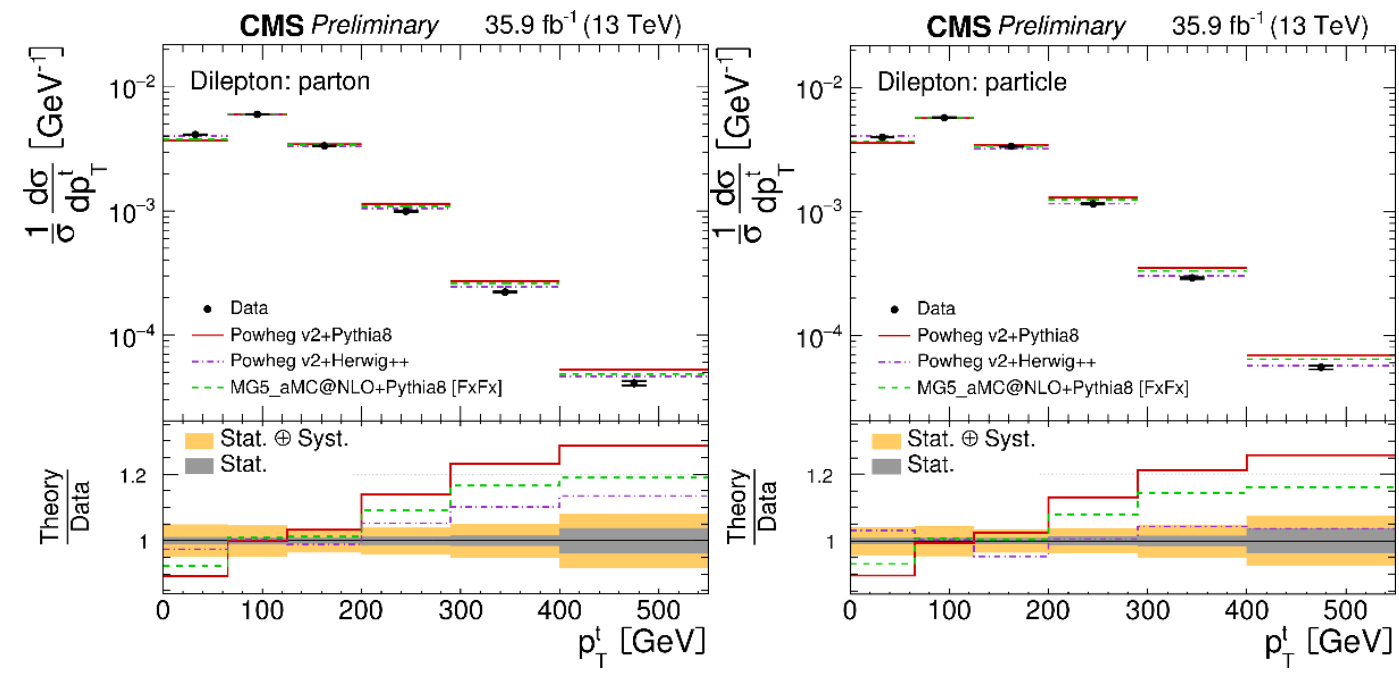

Figure 4. Normalized differential $t \bar{t}$ production cross sections at parton level in full phase space (left) and particle level in fiducial phase space (right) as a function of $\mathrm{p}_{\mathrm{T}}(\mathrm{t})$ as seen in [8]. Several full NLO MC setups interfaced with Pythia8 or Herwig++ for Parton Shower (PS) are shown. The lower panel in each plot shows the ratio of the theoretical prediction to the data.
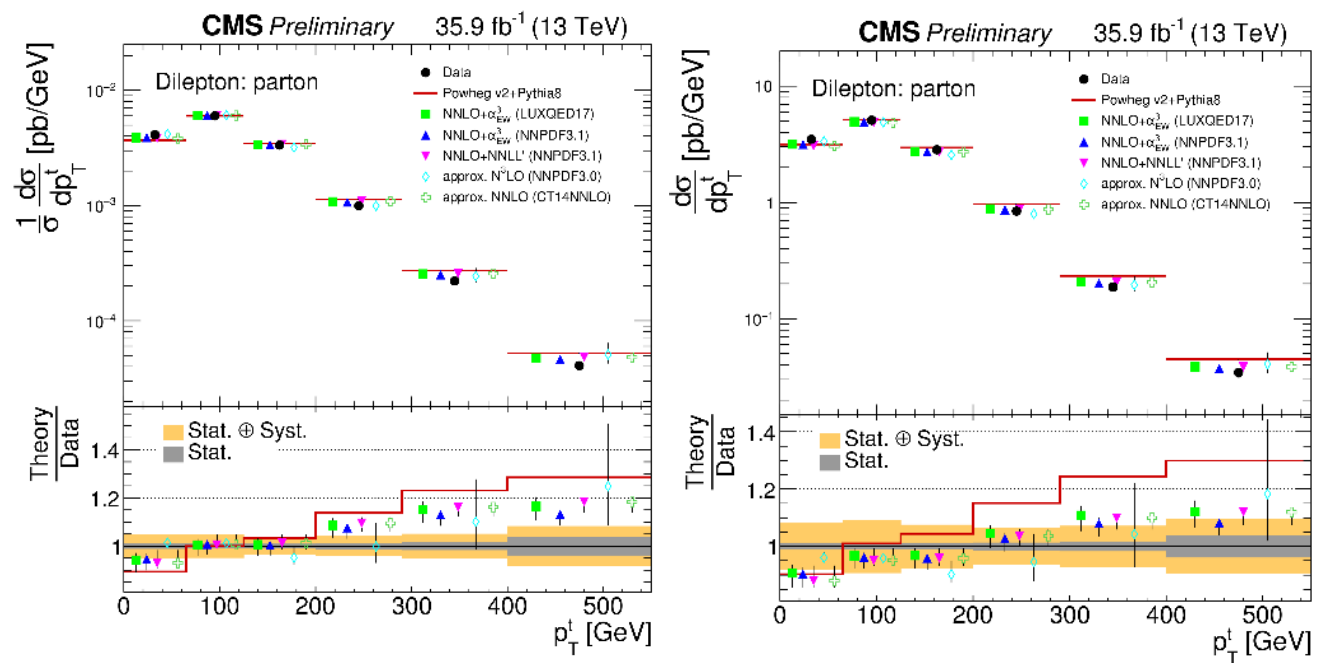

Figure 5. Normalized (left) and non-normalized (right) differential t $\bar{t}$ production cross sections at parton level in full phase space as a function of $\mathrm{p}_{\mathrm{T}}(\mathrm{t})$ as seen in [8]. Several beyondNLO predictions are also shown. The lower panel in each plot shows the ratio of the theoretical prediction to the data.

Other Run2 differential results include: the boosted $\mathrm{p}_{\mathrm{T}}(\mathrm{t})>500 \mathrm{GeV} / \mathrm{c}$ top quark regime [13],

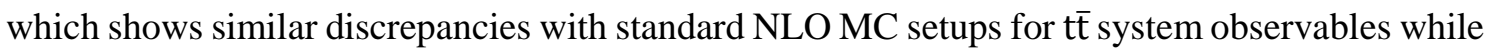
$\mathrm{p}_{\mathrm{T}}(\mathrm{t})$ is mostly acceptable but with large statistical uncertainties; and studies [14,15] of kinematic event variables (i.e. those that do not need a reconstruction of the top quarks using stable particles within experimental acceptance to avoid theory extrapolations) which justify the use of Powheg $\mathrm{V} 2+$ Pythia8 as default generator for $\mathrm{t} \overline{\mathrm{t}}$ processes in the Run2. However, tuned MC setups using Run1 data are needed to reach a reasonable level of agreement in the distributions of observables, which are detector dependent. A strong effort is invested by the LHC experiments trying to use and integrate Herwig 7 [16] as an alternative to Pythia in order to properly quantify the systematic uncertainties in the parton shower interface. 


\section{Double differential cross sections.}

The so called 2D or double differential studies provide more details in corners of the phase space while looking at two observables at the same time. The two latest results from Run2 provide consistent results in the lepton+jets channel: one from CMS [17] using 2016 data and one from ATLAS [18] using 2015 data with results in three independent jet bins but with no 2D unfolding. Both studies show very few discrepancies at parton level but the measurement is dominated by the uncertainty in the parton shower and hadronization modelling. Figure 6 shows the result from CMS where the overprediction of the $\mathrm{p}_{\mathrm{T}}(\mathrm{t})$ is clearly seen specially for the 0 additional jets case which is most sensitive to NLO generator effects; the 1 additional jet bin is really probing the NLO part of the calculation with a smaller effect, while 2 or 3 extra jets have increased systematic uncertainties and are most sensitive to parton shower. Among the different MC setups tested, only Herwig++ seems to have a different trend but it has been superseded by Herwig7. However, no setup predicts all the measured distributions and although the $\mathrm{p}_{\mathrm{T}}(\mathrm{t})$ is in general softer than predicted in all the regions of the phase space, there is a better agreement in the forward regions.
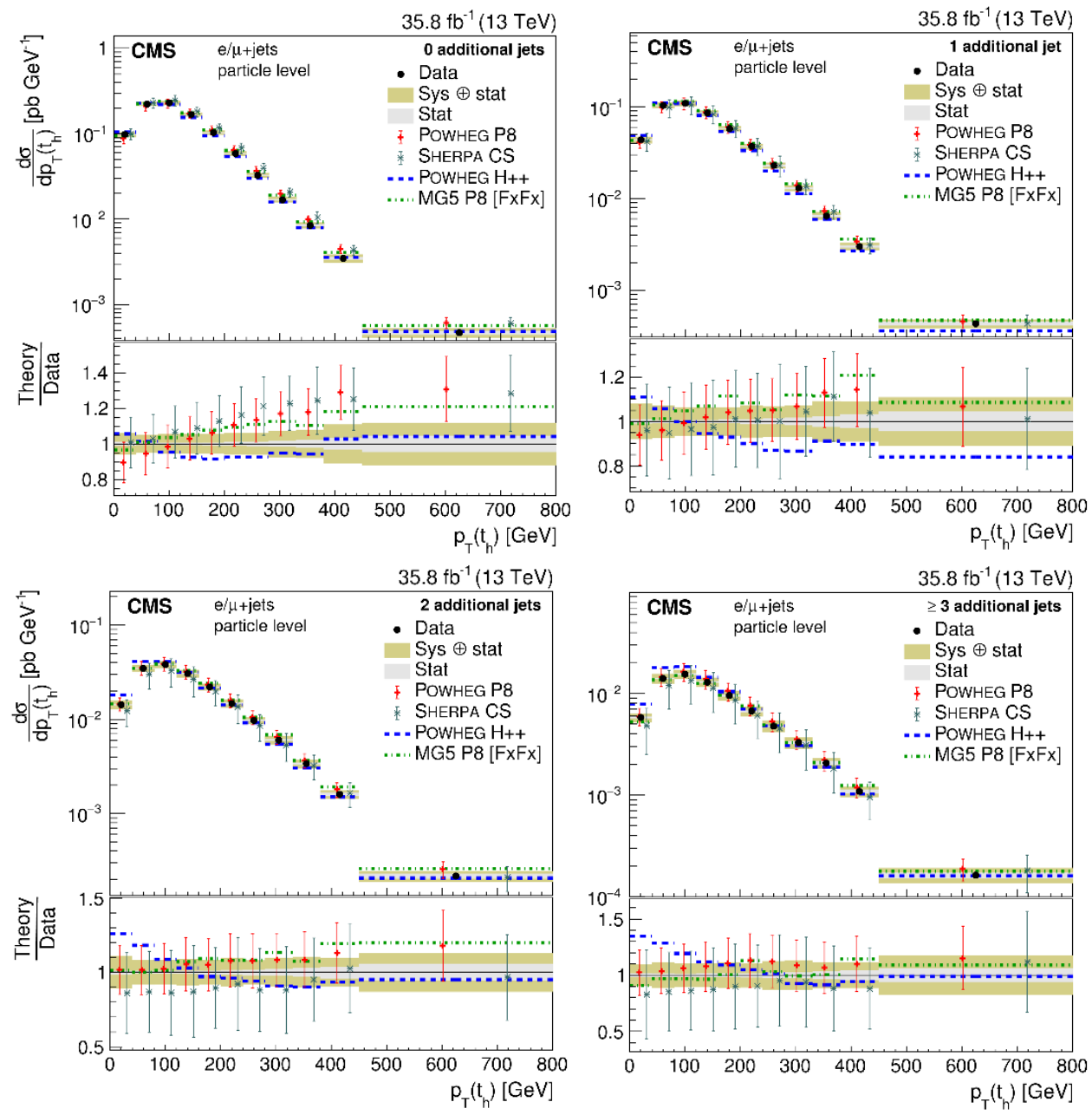

Figure 6. Differential cross sections at the particle level as a function of $\mathrm{p}_{\mathrm{T}}\left(\mathrm{t}_{\mathrm{h}}\right)$ in bins of the number of additional jets [17]. The data are shown as points with light (dark) bands indicating the statistical (statistical and systematic) uncertainties. The cross sections are compared to the predictions of Powheg combined with Pythia8 (P8) or Herwig++ $(\mathrm{H}++)$ and the multiparton simulations MadGraph5_aMCatNLO (MG5)+Pythia8 FxFx [18] and Sherpa [19]. The ratios of the various predictions to the measured cross sections are shown at the bottom of each panel. 


\section{Other differential studies}

Recent LHC Run2 results exploit the sensitivity of the measured cross section to different parameters employed in state-of-the-art MC simulation programs by comparing the results with different simulations. For example, the Underlying Event (UE) study from CMS [21] (Figure 7 left), characterizes, for the first time, the properties of the UE at a factorization scale which is typically above twice the top quark mass. On the other hand, the CMS jet substructure study [22] explores $\sim 15$ physics observables sensitive to the modelling of the system confronting inclusive and flavour-specified jets with a wide array of models (Figure 7 right). Both results provide a compatible scan of the strong coupling constant in final state radiations, $\alpha_{\mathrm{s}}{ }^{\mathrm{FSR}}\left(\mathrm{M}_{\mathrm{z}}\right)$, in UE and jet shapes: $\left\langle\mathrm{p}_{\mathrm{T}}>0.120 \pm 0.006\right.$ and $\lambda_{1}{ }^{1}$ (width) $0.123 \pm 0.001$ respectively. Moreover, an ATLAS study on colour-flow in $t \bar{t}$ events [23] provides normalized fiducial cross sections as a function of pull angle for the hadronically decaying W boson daughters using a wide variety of full MC setups and colour model predictions; while good agreement can be found for some combinations of predictions and observables, none of the predictions describes the data well across all observables.
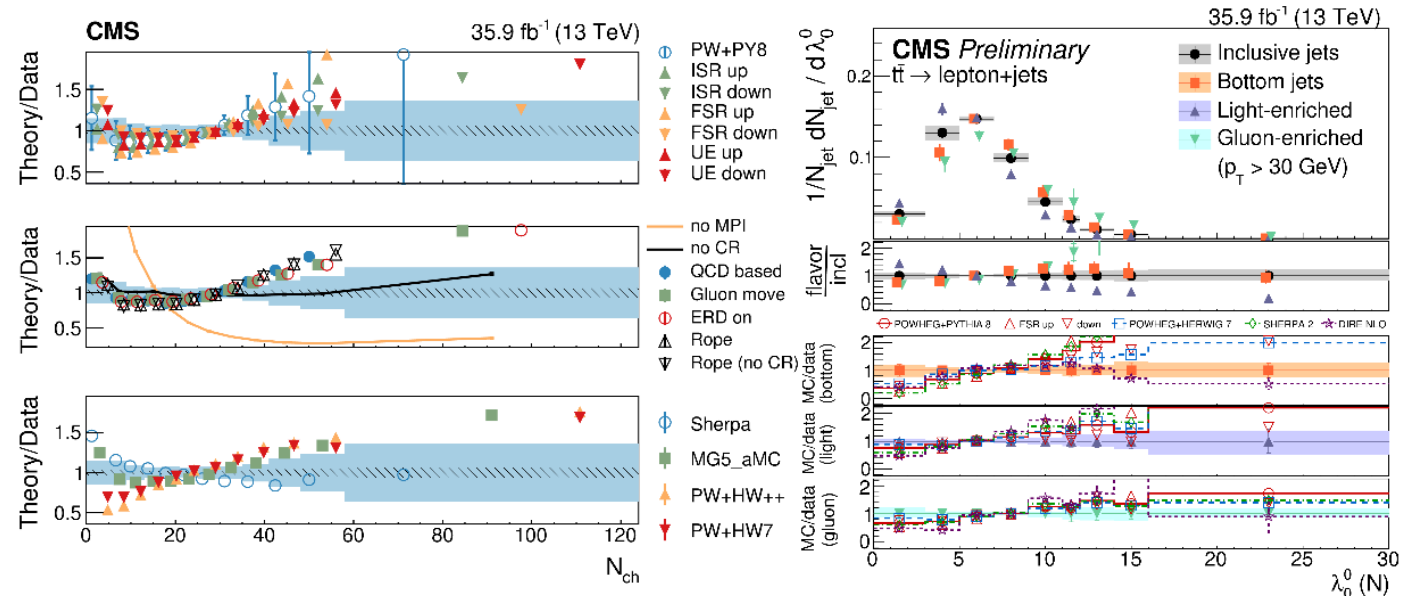

Figure 7. Left: The normalized differential cross section as function of the number of charged particles per event $\left(\mathrm{N}_{\mathrm{ch}}\right)$ in the study on underlying event tunes in $t \bar{t}$ [21]. Right: Distributions of the charged multiplicity per jet $\lambda_{0}{ }^{0}(\mathrm{~N})$ unfolded to the particle level, for jets of different flavors measured in $t \bar{t}$ events for the study on jet substructure observables [22]. Different panels display the ratio between each model tested and the data. In both cases the colored (shaded) band represents the total (statistical) uncertainty of the data, while the error bars represent either the total uncertainty of the Pw+Py8 setup, or the statistical uncertainty of the other MC setups.

\section{Summary and conclusions}

LHC inclusive $t \bar{t}$ cross section results are in good agreement with predictions up to now, including also first results in proton-nucleus $(\mathrm{Pb})$ collisions and from LHCb in the forward region. However, discrepancies have arisen since Run1 when looking at differential distributions, in particular in the full NLO modelling of observables coming from the top and $\mathrm{t} \overline{\mathrm{t}}$ systems. Huge efforts by the ATLAS, CMS and LHCb collaborations are underway to find out what could be missing from our understanding of the $t \bar{t}$ process. A key tool for answering this puzzle are differential 1D/2D cross section measurements. Nevertheless, at present none of the predictions describes the data well across all observables, so observations highlight the need for even more accurate theoretical calculations (perhaps a NNLO+PS prediction or a full NLO Matrix Element + PS treatment), a better understanding of the sources of uncertainty and more data. 


\section{References}

[1] ATLAS Collaboration, The ATLAS Experiment at the CERN LHC, JINST 3 S08003 (2008)

[2] CMS Collaboration, The CMS experiment at the CERN LHC, JINST 3 S08004 (2008)

[3] LHCb Collaboration, The LHCb Detector at the LHC, JINST 3 S08005 (2008)

[4] M. Czakon, P. Fiedler, A. Mitov, The total top quark pair production cross-section at hadron colliders through $O\left(\alpha^{4}\right)$, PRL 110 (2013) 252004 [arXiv:1303.6254]

[5] ATLAS Collaboration, Measurement of the inclusive $t \bar{t}$ cross-section in the lepton+jets channel in pp collisions at $\sqrt{s}=8 \mathrm{TeV}$ with the ATLAS detector, EPJC 78 (2018) 487 [arXiv:1712.06857]

[6] CMS Collaboration, Measurement of the inclusive $t \bar{t}$ cross section in pp collisions at $\sqrt{s}=5.02 \mathrm{TeV}$ using final states with at least one charged lepton, JHEP 03 (2018) 115

[7] CMS Collaboration, Observation of top quark production in pA collisions, PRL119 (2017) 242001

[8] CMS Collaboration, Measurements of differential cross sections for $t \bar{t}$ production in proton-proton collisions at $\sqrt{s}=13 \mathrm{TeV}$ using events containing two leptons,CMS-PAS-TOP-17-014 (Sub. to JHEP)

[9] S. Frixione, P. Nason and G. Ridolfi, Heavy-quark pair production, JHEP 0709 (2007) 126

[10] T. Sjöstrand et al., An introduction to PYTHIA 8.2, Comput. Phys. Commun., vol.191 (2015) 159

[11] M. Bahr et al.: Herwig++ Physics and Manual, EPJC 58:639-707 (2008) [arXiv:0803.0883]

[12] ATLAS Collaboration, Measurements of top-quark pair differential cross-sections in the e $\mu$ channel in pp collisions at $\sqrt{ }_{s}=13 \mathrm{TeV}$ using the ATLAS detector, EPJC 77 (2017) 299

[13] ATLAS Collaboration, Measurements of $t \bar{t}$ differential cross-sections of highly boosted top quarks decaying to all-hadronic final states in pp collisions at $\sqrt{s}_{s}=13 \mathrm{TeV}$, PRD 98, 012003 (2018)

[14] CMS Collaboration, Measurement of the differential cross sections of top quark pair production as a function of kinematic event variables in pp collisions at $\sqrt{s}_{s}=13 \mathrm{TeV}$, JHEP 06 (2018) 002

[15] ATLAS Collaboration, Measurement of jet activity in top-quark events with an electron, a muon and two b-tagged jets in the final state in pp collisions at $\sqrt{s}_{s}=13 \mathrm{TeV}$, EPJC (2017) $77: 220$

[16] J. Bellm et al, Herwig 7.0/Herwig + + 3.0 release note, EPJC76 (2016) 76 : 196

[17] CMS Collaboration, Measurement of differential cross sections for the production of top quark pairs and of additional jets in l+jets events from pp collisions at $\sqrt{s}=13 \mathrm{TeV}$, PRD 97 (2018) 112003

[18] R. Frederix et al., Merging meets matching in MC@NLO, JHEP 1212 (2012) 061 [arXiv:1209.6215]

[19] T. Gleisberg et al., Event generation with SHERPA 1.1, JHEP 0902 (2009) 007, [arXiv:0811.4622]

[20] ATLAS Collaboration, Measurements of differential cross sections of top quark pair production in association with jets in pp collisions at $\sqrt{s}_{s}=13 \mathrm{TeV}$ using the ATLAS detector, JHEP 10 (2018) 159

[21] CMS Collaboration, Study of the underlying event in top quark pair production in pp collisions at $13 \mathrm{TeV}$, CMS-PAS-TOP-17-015 ; CERN-EP-2018-177 [arXiv:1807.02810] (Sub. to EPJC)

[22] CMS Collaboration, Measurement of jet substructure observables in $t \bar{t}$ events from pp collisions at $\sqrt{s}_{s}=13 \mathrm{TeV}$, PRD 98, 092014 (2018) [arXiv:1808.07340]

[23] ATLAS Collaboration, Measurement of colour flow using jet-pull observables in $t \bar{t}$ events with the ATLAS experiment at $\sqrt{ }_{s}=13 \mathrm{TeV}$, EPJC 78 (2018) 847 [arXiv:1805.02935] 